\title{
UNA REFLEXIÓN ACERCA DE LAS TUTORÍAS UNIVERSITARIAS A PARTIR DE LAS VALORACIONES REALIZADAS POR PROFESORES /TUTORES DE LA UNIVERSITAT DE VALÈNCIA
}

\author{
A REFLECTION ABOUT UNIVERSITY TUTORSHIP FROM THE \\ ASSESSMENT OF UNIVERSITY OF VALENCIA PROFESSORS
}

\author{
Inmaculada Chiva Sanchis* y Genoveva Ramos Santana*
}

Universitat de València

\section{RESUMEN}

Este artículo responde a la necesidad de reflexionar acerca de las tutorías universitarias para alumnos de primero y sobre cómo llevar a cabo, de la manera más eficaz y efectiva posible, el Plan de Acción Tutorial (PAT). Esta reflexión se realiza a partir de las consideraciones, aportaciones y experiencias personales que algunos profesores/tutores han manifestado a lo largo de distintas sesiones de trabajo respecto a la implementación del PAT en la Universitat de València. Todo ello, con el objetivo de mejorar e innovar en el contexto universitario basándonos en experiencias reales.

Palabras claves: Universidad. Tutorías Universitarias. Estudiante Universitario. Formación del tutor. Plan Tutorial.

\begin{abstract}
This article responds to the need of reflecting about university tutoring for first year students and how to accomplish the Tutorial Action Plan (TAP) in the most effective way. This reflection is real, emerging from the considerations, contributions and personal experiences that some professors and tutors have manifested along some work sessions in relation to the implementation of the TAP at the University of Valencia. All of it, with the aim of enhancing our practice and innovating in the university context based on real experiences.
\end{abstract}

\footnotetext{
* Inmaculada Chiva Sanchis, E-mail: inmaculada.chiva@uv.es y Genoveva Ramos Santana, E-mail: genoveva. ramos@uv.es son doctoras en Filosofía y Ciencias de la Educación desarrollan su actividad investigadora dentro del Grupo de Evaluación y Medición (GEM-www.uv.es/gem) de la Universitat de València, coordinado por el Dr. D. Jesús M. Jornet Meliá. Participan en las siguientes líneas de trabajo: Evaluación de la Docencia Universitaria, de Centros, de Programas para el Empleo, de Programas de Teleformación, y de Sistemas Educativos.
} 
Key words: University. Higher Education. University Student. Tutor Training. Tutorial Plans. Tutoring.

\section{Introducción}

Una de las consecuencias más directas en los proyectos de innovación para la creación de un Espacio Europeo de Educación Superior ha sido la reducción de las clases magistrales por el trabajo dirigido y autónomo del estudiante como parte fundamental de su formación. Parece necesario, pues, establecer metodologías que nos acerquen más al estudiante de forma individual, a sus aprendizajes y a sus limitaciones y carencias. Entre dichas metodologías nos encontramos con las tutorías (Salinas y Cotillas, 2004) que en los últimos años han suscitado un interés por parte de las universidades.

De este interés incipiente por las tutorías universitarias surge este artículo y concretamente de las reflexiones a las que hemos llegado tras la impartición de los cursos de formación: "Tutorías: Seguimiento de la tutorización de los alumnos universitarios", por parte de las autoras, desarrollados para el Servei de Formació Permanent de la Universitat de València durante el curso académico 2004-2005. Estos cursos tenían como objetivo proporcionar información y elementos suficientes para que cada docente diseñara, planificara y desarrollara un Plan de Acción Tutorial acorde con sus posibilidades y limitaciones. Asimismo, estaban dirigidos a todos los tutores encargados de orientar a los estudiantes sobre todo de los primeros cursos, en grupos concretamente de innovación docente.

\section{Objetivos del artículo}

Con la elaboración de este artículo pretendemos reflexionar acerca de las posibilidades y limitaciones que ofrece el desarrollo de un Plan de Acción Tutorial (PAT) para los estudiantes de primero dentro de los proyectos de innovación docente, suscitadas éstas a partir de los debates que se establecieron entre los tutores asistentes a los cursos de "Tutorías: Seguimiento de la tutorización de los alumnos universitarios". Este objetivo general nos conduce a establecer dos objetivos específicos:

1. Plantear el Plan de Acción Tutorial Base a partir del cual analizar y reflexionar sobre sus posibilidades y limitaciones a la hora de aplicarlo.

2. Extraer algunas conclusiones apoyadas en la experiencia propia y en la de profesores/ tutores de estudiantes de primero que participaron en los cursos, con el fin de contribuir a la mejora del desarrollo e implantación de las tutorías en la Universidad.

\section{El plan de acción tutorial}

Antes de comenzar a explicar las características que definirían el PAT es importante aclarar ciertos aspectos teóricos relacionados. Entendemos por tutoría aquella actividad docente que tiene una metodología didáctica específica y que se desarrolla en distintas sesiones entre un profesor universitario y uno o varios estudiantes, con el fin de ayudarlos y 
orientarlos en su evolución académica, vocacional y personal, lo que permite al mismo tiempo que el profesor mejore en su intervención docente con los estudiantes (Salinas y Cotillas, 2004; Rodríguez Espinar, 2004; Almajano, 2003; Pérez Boullosa y Blasco, 2002; Blasco, (s.f.)).

Por otro lado, el tipo de tutorías a las que nos referimos en el PAT, que presentaremos a continuación, se ajusta a una tutoría de orientación, asesoramiento o asistencial dirigida a estudiantes de primero (de transición), cuyo responsable más directo es el profesor/tutor y que se desarrolla en un tramo formativo concreto con sesiones tanto individuales como grupales.

De ahí que entendamos que al igual que seguimos un proceso para la elaboración de nuestra práctica docente hemos de desarrollar un proceso metodológico de nuestra acción tutorial, puesto que es necesario delimitar, previamente a la acción, todos los elementos que van a intervenir en la misma. Es decir, se trata de que generemos una secuencia con pautas determinadas de asesoramiento y seguimiento de los estudiantes tutorizados en un periodo determinado, de manera que existan muy pocos elementos expuestos a la improvisación. Teniendo en cuenta las aportaciones realizadas por el Servei de Formació Permanent de la Universitat de València ${ }^{1}$, la revisión de literatura existente sobre este tema (Almajano, 2003; Enguita, 2003; Rodríguez Espinar, 2004; Gairín et al., 2004 y Román, 2004) y los materiales de apoyo para la realizar la función tutorial "cuaderno del profesor-tutor"de la Universidad Politécnica de Valencia (UPV) ${ }^{2}$, decidimos plantear una propuesta de Plan de Acción Tutorial para estudiantes de primero en los cursos de formación especificados anteriormente que nos permitiera reflexionar, plantear dudas, aspectos positivos y negativos... junto con los tutores asistentes a los cursos. De este modo reflexionamos sobre:

\section{a) Los Objetivos del PAT}

Entre los objetivos que el PAT debe cubrir decidimos plantear dos niveles de concreción de los mismos: generales y operativos. Como generales: Facilitar la transición y la integración a la vida universitaria; Mejorar el rendimiento académico; Conocer en mayor medida a los estudiantes; Mejorar en el desarrollo profesional del docente; Y alcanzar calidad educativa universitaria. Como operativos: proporcionar información sobre aspectos sociales del contexto universitario (con la intención de desarrollar su autonomía personal); proporcionar a los estudiantes estímulos para la reflexión y el diálogo en el ámbito académico; proporcionar información sobre aspectos administrativos; proporcionar información sobre aspectos académicos (enseñar aprender a aprender).

1. "Guía de Tutors de Primer Curs. Tutories per a la Transició" e "Introducció a les Tutoríes. Materials per a la Reforma".

2. La necesidad de potenciar la función tutorial del profesorado en la UPV surge con el llamado Proyecto Europa cuya intención es mejorar la calidad de la educación tanto en factores vinculados a la enseñanza como al aprendizaje. Este proyecto se pone en marcha durante el curso académico 2000/2001 por el Vicerretorado de Coordinación Académica y Alumnado (García, E., Cáceres, P. y Rodríguez, C., 2003). 


\section{Consideraciones aportadas por parte de los tutores asistentes a los cursos ${ }^{3}$}

En cuanto, a los objetivos generales planteados, los tutores asistentes al mismo nos matizaron que las tutorías tienen como objetivo "conocer en mayor medida a los estudiantes" puesto que "sirven para que los estudiantes se conozcan y para que nosotros como profesores de primero conozcamos a los estudiantes". Asimismo, señalan como importante el "facilitar la transición y la integración a la vida universitaria del estudiante de primero", aunque matizan - y aquí hemos de destacar que lo hacen de una manera muy reiterada- que no se trata de "llevarlos de la mano" sino solamente ayudarles en los aspectos académicos, administrativos y sociales del propio contexto universitario. Casi el cien por cien de los profesores asistentes, a este curso, persisten en la idea de no restar autonomía e independencia a los estudiantes cuando llegan a la universidad, ya que piensan que estos están cansados del control existente en las tutorías de los niveles educativos anteriores. Además, consideran que ciertos objetivos como: mejorar el rendimiento académico, mejorar en el desarrollo profesional y aumentar la calidad educativa en la Universidad son inalcanzables, por lo menos por ahora, ya que dependen de factores que superan a la tutoría.

\section{b) Los Contenidos del PAT (contenidos conceptuales, procedimentales y actitudinales)}

Contenidos conceptuales ("saber"). Los estudiantes necesitan de estos conocimientos porque les resultan útiles para resolver tareas o problemas de forma precisa en su proceso de integración y formación académica y personal dentro de la institución (información sobre distintas asignaturas y departamentos; particularidades de los planes de estudio - optatividad, libre elección, vías de acceso, salidas profesionales...-; información sobre normativas académicas generales, etc.).

Contenidos procedimentales ("saber hacer"). Una de las características de estos contenidos es que se consolidan con la práctica, por ello, demandan un contexto activo de aprendizaje, aspecto importante en todo el proceso de acción tutorial. Entre estos contenidos nos podemos encontrar con el conocimiento sobre los distintos recursos tecnológicos, estrategias y técnicas pedagógicas; contacto con las asociaciones de estudiantes y la organización social de la propia facultad, contacto con la comunidad científica que genera y controla las nuevas aportaciones en el ámbito.

Contenidos actitudinales ("saber ser"). Las actitudes se manifiestan a través de comportamientos y expresan preferencias o elecciones manifestadas ante hechos concretos. Entre ellos nos encontramos con: los tipos de relaciones estudiante/ profesor; reconocer el valor/utilidad de los conocimientos y de su aprendizaje; presentar el altruismo como valor y como meta de todo esfuerzo; desarrollar la creatividad e imaginación en el diseño y presentación de los trabajos; ...

3. Consideraciones extraídas de las actividades realizadas con los grupos de profesores/ tutores durante el curso "Tutorías: Seguimiento de la tutorización de los alumnos universitarios", las cuales se van a presentar en todos los apartados del PAT. 


\section{Consideraciones aportadas por parte de los tutores asistentes a los cursos}

En cuanto a los contenidos, los profesores/tutores asistentes al curso, especifican de manera muy concreta que ellos se dedican más a contenidos conceptuales y menos a los procedimentales y actitudinales. Esto se debe a que el estudiante llega, a las tutorías, con dudas referentes a asignaturas, departamentos, planes de estudios, salidas profesionales, ... De ahí surgió una reflexión importante, "estaría bien que no nos basáramos sólo en contenidos conceptuales sino que fuéramos un poco más allá, a procedimientos y actitudes", lo cual sólo conseguiremos si planteamos el PAT como un todo organizado y planificado, donde hayan sesiones de enseñanza por parte del tutor, sesiones de dudas por parte del estudiantes y sesiones de evaluación de lo que estamos realizando.

Por otro lado, otro problema detectado respecto a los contenidos es, cómo saber todo acerca de lo que se consulta. Aquí los tutores concluyen que no es necesario saber "todo" puesto que lo importante es orientar al estudiante en la resolución de problemas. Por último, otra reflexión que surgió al exponer este tema es que los estudiantes no están tan mal informados aunque quizás la información que poseen no sea la más cercana a la realidad, lo que puede generar problemas y dificultades en cualquier momento.

\section{c) La Metodología del PAT}

Desde una perspectiva centrada en el estudiante, creemos que desde el punto de vista metodológico en la tutoría el profesor debe actuar como guía u orientador, tomando como punto de partida las características personales de los estudiantes y elaborar desde ahí el diseño de trabajo a realizar en las distintas sesiones. Además, creemos que se deben analizar cada una de las variables imprescindibles en el desarrollo metodológico de la acción tutorial como por ejemplo: los escenarios (despacho del tutor, sala de reuniones, ...), los métodos, los materiales y los recursos. Por otro lado, un elemento fundamental desde el punto de vista metodológico es la motivación - tanto del estudiante como del tutor- como variable que interviene en el proceso de cualquier comportamiento organizado, regulándolo de acuerdo con los fenómenos ambientales, la situación y los objetivos personales.

\section{Consideraciones aportadas por parte de los tutores asistentes a los cursos}

En cuanto a la metodología, la mayoría de tutores coinciden en la necesidad de motivar al estudiante, puesto que es uno de los mayores problemas encontrados "estudiantes desmotivados y apáticos, casi obligados a venir". Por lo que se hace imprescindible para desarrollar de manera adecuada este proceso, intentar activar la curiosidad-atención de los estudiantes, siendo necesario llegar a conocer y comprender al estudiante que tenemos delante y de este modo llegar a sus intereses y necesidades (ya que sólo desde aquí podemos motivarles para que aprendan a aprender). Asimismo, otro aspecto destacado fue la heterogeneidad de los grupos de estudiantes de un tutor, por lo que se ve como conveniente utilizar una metodología más individualizada en las tutorías y en menor porcentaje las grupales.

Por último, no hay que olvidar, tal y como se comentó durante las sesiones del curso, que los profesores /tutores asistentes diferencian de manera clara entre tutorías y terapia. La tutoría no es una terapia sino que su objetivo es asesorar y/o motivar a los estudiantes. 
Por lo que sería conveniente recordar que, aunque a veces estrecha, hay una línea que separa la tutoría de la terapia. De ahí que siempre que se detecte un problema que supere los objetivos del PAT es necesario derivar hacia gabinetes/organismos donde el estudiante puede recibir la ayuda especializada.

\section{d) Los Recursos y Materiales del PAT}

Cuando hablamos de recursos y materiales en el proceso de acción tutorial nos encontramos con elementos que a nuestro modo de entender son imprescindibles: fichas y cuestionarios de recogida de información por un lado, y, por otro, documentación en papel o electrónica para proporcionar al estudiante. Por ejemplo: Ficha Personal del estudiante + Cuestionario individual de recogida de información inicial (al inicio de la acción tutorial); Ficha de seguimiento de tutorías individuales + Entrevistas semiestructuradas individuales (durante el desarrollo de la acción tutorial); Fichas de seguimiento de tutorías grupales (durante el desarrollo de la acción tutorial); Fichas de seguimiento individual de evaluaciones (durante el desarrollo de las tutorías); Cuestionario individual de recogida de información final (al final de la acción tutorial); y Entrevista semiestructurada de recogida de información grupal (al final de las tutorías).

Sobre los recursos merece la pena hacer una especial mención a las nuevas tecnologías de la información y la comunicación. Las tutorías se realizan mayoritariamente de forma presencial pero también es fundamental la página web del profesor, donde éste "colgará" la información y documentación que estime oportuna, así como, el correo electrónico y el chat.

\section{Consideraciones aportadas por parte de los tutores asistentes a los cursos}

Aquí pudimos comprobar que la mayoría de los tutores carecían de recursos propios y organizados, encontrándose exclusivamente en algunos casos, con "el cuaderno de acción tutorial" de alguna facultad específica. La mayoría no se habían planteado elaborar ni diseñar los materiales necesarios puesto que se encontraban a la demanda del estudiante. Sólo muy pocos utilizaban su página web como recurso de comunicación con el estudiante, colgando la documentación y la información necesaria para este.

\section{e) La Planificación o Secuenciación de las reuniones en el PAT}

Es un hecho comprobado que la planificación de las reuniones y su conocimiento reducen la ansiedad tanto por parte de los profesores como de los estudiantes, ya que desciende el nivel de ambigüedad y de incertidumbre durante el proceso instruccional. Se hace necesario preparar una planificación de las reuniones con los estudiantes incluyendo: número de sesiones "necesarias" con horario estimado según el/los tema/s a tratar; guión que presente los puntos principales de cada sesión; diseño previo de todo el contenido y recursos a impartir en cada sesión; diseño previo del tipo de actividad a realizar en cada sesión, anticipando dichas actividades; estructura de las sesiones que favorezca la participación y el intercambio de información ("doy y recibo") ... 


\section{Consideraciones durante el curso por parte de los tutores}

Tras las conversaciones y el trabajo en grupo, los profesores /tutores comentan que llevan a cabo las sesiones establecidas de forma institucional, pactándose con el estudiante las que este necesite de más. Detectamos que en general las sesiones institucionalizadas eran un mero trámite de recogida de información, mientras que si el estudiante necesitaba alguna información específica ya se pactaba con él una nueva reunión a tal efecto, por lo que se podrían denominar "sesiones a la demanda". Por otro lado, también se trató el tema de la realización de sesiones individuales o grupales en las tutorías llegando a la conclusión que dependen del objetivo de las mismas y del tipo de estudiante.

\section{f) La Evaluación del PAT}

La evaluación constituye un proceso dirigido de recogida de información relevante cuya finalidad es informar en los procesos de toma de decisiones. Partiendo de esta premisa creemos que la finalidad de la evaluación del PAT es doble, por un lado, mejorar su eficacia y su eficiencia, es decir, hemos de llevar a cabo un análisis del Plan con la finalidad de optimizar los recursos del mismo orientados a la consecución de los objetivos previstos (eficiencia) e incrementar los logros del Plan (eficacia). Y por otro lado, optimizar la calidad de la acción tutorial universitaria. De ahí la necesidad de desarrollar un proceso de evaluación basado en las opiniones de los estudiantes, desarrollado en distintos momentos. Los momentos serían los siguientes: una evaluación inicial a través del pase de cuestionarios de recogida de información inicial y general de cada estudiante o de las fichas individuales de los mismos. Una evaluación continua del plan, durante su desarrollo, mediante las fichas de seguimiento individuales y grupales, análisis del sistema de aprendizaje del estudiante, el control de asistencia, la motivación y la actitud del estudiante en las sesiones, etc. Y por último, una evaluación de resultados, al final de la acción tutorial, es decir, conocer el grado en que se han conseguido los objetivos para los que se diseñó el Plan. Al mismo tiempo, este proceso de evaluación ha de completarse recogiendo las opiniones de nuestros colegas tutores, buscando la forma de triangular la información recogida a partir de la opinión de los estudiantes. Así, por ejemplo, se podrían organizar reuniones periódicas con tutores que estén desarrollando un Plan de Acción Tutorial en la misma facultad.

\section{Consideraciones durante el curso por parte de los tutores}

Con respecto a esta fase pudimos comprobar como los profesores/ tutores asistentes al curso no habían desarrollado todavía ningún proceso de evaluación. Del mismo modo que tampoco llevaban a cabo procesos de autoevaluación. De ahí, la necesidad que manifestamos en su día respecto al desarrollo de la evaluación como sistema de retroalimentación de su propia acción como tutores.

\section{Conclusiones}

Para terminar sintetizamos las necesidades generales que los tutores plantearon a la hora de desarrollar cada Plan de Acción Tutorial. Destacándose entre ellas las siguientes: 
- Tiempo, gracias al cual se mejoraría la gestión del plan, la organización, la planificación de las sesiones y los materiales. Puesto que cuando no se dispone del mismo la acción tutorial se vuelve desmotivadora, poco eficaz, burocrática y se convierte en una mera recogida de información para satisfacer objetivos institucionales ${ }^{4}$.

- Mayor formación específica respecto al tema de las tutorías. Se demandan cursos teórico-prácticos donde puedan adquirir los aprendizajes requeridos para el desempeño de su labor como tutores.

- Materiales, llámese "cuaderno del tutor", adecuados para sus necesidades y adaptados a cada una de las facultades, con el objetivo de poder utilizarlo/s como herramienta/s de ayuda, de guía para organizar los aspectos a tratar y con la opción de poder personalizarlo según las necesidades de los estudiantes y el criterio del tutor.

- Especificidad de las funciones del tutor, los tutores manifiestan tener muy claras las "no funciones del tutor": hacer de terapeuta, aparentar ser amigo de los estudiantes o dar clases particulares de las asignaturas... aunque no tienen tan claras las verdaderas funciones específicas.

- Coordinación entre tutores, para poder realizar una evaluación continua del desarrollo del PAT, poder subsanar errores más rápidamente e ir aprendiendo de la experiencia compartida. Por otro lado, es importante conocer también los resultados que a nivel institucional nos pueda aportar la Universidad para comprobar si los objetivos de las Tutorías se han cumplido, si es necesario replantear el PAT, qué aspectos se tendrían que mejorar, etc.

\section{Referencias bibliográficas}

Almajano, M". P. (2003). "La tutoría académica universitaria en la sociedad del conocimiento: un elemento de calidad y de mejora para nuestros estudiantes". En Álvarez Pérez, P. y Jiménez Betancort, H., Tutoría Universitaria. Colección Documentos Congresuales. Santa Cruz de Tenerife. Servicio de Publicaciones: Universidad de la Laguna.

Blasco, P. (s.f.). Proyectos de Innovación en Tutorias. Orientación para la transición entre la educación secundaria y la Universidad. [http://www.uv.es/ sfp/pdi/DocTransic.pdf, consulta del 27-10-2005].

Enguita, C. (2003). "Plan tutorial de la Universidad Europea CEES". En Álvarez Pérez, P. y Jiménez Betancort, H. Tutoría Universitaria. Colección Documentos Congresuales. Santa Cruz de Tenerife. Servicio de Publicaciones. Universidad de la Laguna.

Gairín, J., Feixas, M., Franch, J., Guillamón, C. y Quinquer, D. (2004). "Elementos para la elaboración de planes de tutoría en la universidad". Contextos educativos 6-7: 21-42.

García, E., Cáceres, P. y Rodríguez, C. (2003). "Materiales de apoyo para realizar la función tutorial 'Cuaderno del Profesor-Tutor de la UPV' ’, en Álvarez Pérez, P. y Jiménez Betancort, H., Tutoría Universitaria. Colección Documentos Congresuales. Santa Cruz de Tenerife. Servicio de Publicaciones: Universidad de la Laguna.

4. Estos objetivos institucionales venían marcados por la propia convocatoria realizada por la Universitat de València para poder incluir las Tutorías de estudiantes de primero dentro de los Proyectos de Innovación de las titulaciones 
Pérez Boullosa, A. y Blasco, P. (2002). Orientación e inserción profesional: fundamentos y tendencias. Valencia: Nau Llibres.

Rodríguez Espinar, S. (Coord.) (2004). Manual de tutoría universitaria. Ed. Octaedro/ ICE-UB.

Román, J. M. (2004). "Modelo 'CARI' de tutoría de estudiantes en la Universidad: procedimiento de formación de profesores mediante 'Reflexión en grupo sobre la práctica'”. Contextos educativos 6-7: 43-64.

Salinas, B. y Cotillas, C. (2004). La tutoria universitària per als estudiants de primer curs. Tutories per a la transició. Servei de Formaciò Permanent. Universitat de València.

Fecha de recepción: 30-11-05

Fecha de revisión: 16-09-07

Fecha de aceptación: 07-11-07 